\title{
Basal Ganglia Hemorrhage: Minimally Invasive and Neuro-Navigation Guided Treatment by Injection of Recombinant Tissue-Type Plasminogen Activator (rtPA)
}

\author{
Ntoulias G, Maslehaty H, Petridis AK*, Bitter A, Morina D, Niklewski F, Hassler W and Scholz M \\ Department of Neurosurgery, Klinikum Duisburg, Academic Teaching Hospital of University Essen-Duisburg, Germany
}

"Corresponding author: Athanasios K. Petridis, Department of Neurosurgery, Klinikum Duisburg, Academic Teaching Hospital of University Essen-Duisburg, Zu den Rehwiesen 9-11, 47055 Duisburg, Germany, Tel: +49 203 733-0; E-mail: opticdisc@aol.com

Rec date: Mar 14, 2014, Acc date: Apr 16, 2014, Pub date: Apr 18, 2014

Copyright: @ 2014 Ntoulias G, et al. This is an open-access article distributed under the terms of the Creative Commons Attribution License, which permits unrestricted use, distribution, and reproduction in any medium, provided the original author and source are credited.

\begin{abstract}
Objective: Since open surgical evacuation of basal ganglia hemorrhage (BGH) as had been proved did not lead to the desired benefit for the patients, we used minimally invasive clot lysis by injection of recombinant tissue-type plasminogen activator (rtPA).

Methods: Patients with spontaneous BGH with or without accompanied intraventricular hemorrhage were included in the study. Following diagnosis of BGH by CT scanning, a catheter was inserted through a small burr hole under neuro-navigation assistance into the centre of hemorrhage and three mg rtPA was injected for three consecutive days. CT scans were performed daily to determine the volume of the hemorrhage. The size of hemorrhage was calculated using the ABCs measuring method. The clinical condition of the patients was classified using the Glasgow coma scale (GCS) and Glasgow outcome scale (GOS). Statistical analysis was performed using the Fisher's exact test and Chi-square with Yates' correction. The p-values were calculated with two tails and statistical significance was estimated as p-value $<0.05$.
\end{abstract}

Results: The procedure was performed on 22 patients (11 females and 11 males). The mean age of the patients was 64 years (range: $44-80$ years). Among the patients, $7(31.8 \%)$ were considered in a poor clinical condition, 11 $(50 \%)$ in an intermediate condition and $4(18.2 \%)$ in a good clinical condition. The total mortality was calculated with $\mathrm{n}=3(13.64 \%)$. The mean volume of hemorrhage before treatment was $35.14 \mathrm{~mL}$ (range $11.5 \mathrm{~mL}-67.15 \mathrm{~mL}$ ). After the third injection, a mean volume reduction of $73.05 \%$ could be achieved. Considering the accuracy of the neuronavigation dataset, correct placement of the drainage in the assumed center of hemorrhage was confirmed in all cases by CT scanning after the first injection. Re-bleeding occurred in 4 cases $(18.2 \%)$.

Conclusion: Treatment with rt-PA in BGH seems an easy, safe and minimally invasive alternative to open surgical hematoma evacuation and it might be more favorable compared to conservative treatment. In our study, we achieved a mean volume of hemorrhage reduction of $73 \%$ after 3 days. Clot lysis alone was not the only predictive factor for a better outcome. Outcome prognosis should take several other co-factors into account such as the age of the patient and the GCS at the time of Admission. Large prospective and randomized trials are still necessary to answer these open questions.

Keywords: Intracerebral hemorrhage; Clot lysis; Recombinant tissue-type plasminogen activator; Minimally invasive; Basal ganglia hemorrhage

\section{Introduction}

Spontaneous intracerebral hemorrhage (ICH) is a severe medical condition associated with complex neurofunctional morbidity which affects about 10-20 per 100,000 people every year. The level of mortality from ICH is higher compared to cerebral ischemic stroke, and the majority of deaths occur within the first days after the hemorrhage [1-22].

Effective treatment of ICH is still an unsolved and debatable issue. Even the large STICH (Surgical Treatment of Ischemic Heart Failure) trial failed to bring clarification. Although the authors of the STICH study concluded no significant benefit from early surgery, compared with initial conservative treatment, it should be noted that about $25 \%$ of the patients were treated surgically a few days after randomization due to neurological deterioration [16,18]. Despite the results of this trial, several institutes prefer the early surgical strategy before deterioration in lobar ICH $[15,18]$.

Different studies have shown that open surgical evacuation does not lead to the desired benefit for patients with spontaneous hemorrhage in the basal ganglia (BGH), when compared to conservative treatment. Moreover, surgical procedures may increase the morbidity due to direct damage of brain structures [8]. Hence, minimally invasive treatment techniques arise as alternative surgical models for deep seated ICH with the goal of gently reducing the hematoma size and preventing secondary damage to the surrounding brain tissue $(5,9,17,23-31]$.

Our aim was to study the application of minimally invasive, neuronavigation guided clot lysis by injection of recombinant tissue-type 
Citation: Ntoulias G, Maslehaty H, Petridis AK, Bitter A, Morina D, et al. (2014) Basal Ganglia Hemorrhage: Minimally Invasive and NeuroNavigation Guided Treatment by Injection of Recombinant Tissue-Type Plasminogen Activator (rtPA). J Neurol Disord 2: 158. doi: $10.4172 / 2329-6895.1000158$

Page 2 of 5

plasminogen activator (rtPA) in patients suffering from spontaneous BGH.

\section{Methods}

\section{Patients and grading systems}

In this study we included 22 patients (11 males and 11 females) suffering from spontaneous BGH with or without accompanying small intraventricular hemorrhage. The mean age of the patients was 64 years (age range: $44-80$ years). The diagnosis was made by CT scanning within 24 hours after onset for all cases. The clinical conditions of the patients at the time of admission was classified using the Glasgow coma scale (GCS) and at time of discharge the Glasgow outcome scale (GOS) The median hospital stay duration among patients with BGH was 22 days. To simplify the results and to further analyse the clinical condition of the patients, the subjects were divided into three sub-groups: poor (GCS $<8$ ); intermediate (GCS 9-12) and good (GCS $>13$ ). The GOS grades were summarized into 3 groups with poor (GOS 1 and 2), intermediate (GOS 3 ) and good outcome (GOS 4 and 5). The size of the hemorrhage was calculated using the $A B C$ method. As explained by Kothari et al. [12]. A is defined as the greatest hemorrhage diameter on the CT scans, B is the diameter 90 degrees to $\mathrm{A}$, and $\mathrm{C}$ is defined as the number CT slices in which the hemorrhage is visible multiplied by the slice thickness. Only patients with hemorrhages $>10 \mathrm{~mL}$ were included in this trial. Patients with $\mathrm{BGH}<10 \mathrm{ml}$ were generally treated conservatively. On the other hand BGHs which reach the cortical surface were usually treated with an open microsurgical trepanation. Patient who underwent open surgery for hematoma evacuation in line with re-bleeding after rtPA Lysis were excluded of this study. To analyse the hematoma reduction, we created four sub-groups: volume reduction $<25 \%, 25-50 \%, 51-75 \%$ and $>75 \%$.

\section{Procedure}

Pre-operative CT datasets (axial CT scans) were obtained using the Brain Lab intraoperative guidance system (Brain Lab ${ }^{\circ}$ ). The head of the patient was fixed straight in a Mayfield frame. A burr hole $(5 \mathrm{~mm}$ diameter) was applied left or right frontal, $2 \mathrm{~cm}$ laterally to the sagittal suture at the coronal suture level. Afterwards a flexible catheter (Codman drainage $e^{\oplus}, 3 \mathrm{~mm}$ in diameter, $1.5 \mathrm{~mm}$ lumen) was fixed at the neuro-navigation pointer and was introduced slowly under position control to the center of hemorrhage. The pointer was removed and the catheter was fixed on the skin. The patients were treated in the intensive care unit (ICU) postoperatively and received standard therapy and monitoring for ICH. Three mg rtPA was injected once a day through the catheter for three consecutive days. After injection of rtPA through the catheter, the catheter was closed and was re-opened after 2 hours. The catheter was connected with a special external CSF drainage system in order for the blood clot to drain off. A blood aspiration does not take place in this procedure. CT scans were performed daily to determine the result of the therapy. The dose of 3 $\mathrm{mg}$ of rt-PA was used according to the procedures in the literature, in which low dose rt-PA application showed good results. This is an established treatment strategy since the first results published by Schaller et al. [19].

\section{Statistical analysis}

Statistical analysis was performed using the Fisher's exact test and Chi-square with Yates' correction using the online calculator on http:// vassarstats.net. Statistical significance was estimated with a two-tailed p-value $<0.05$.

\section{Results}

Twenty two patients (11 females and 11 males) aged from 44 to 80 years (mean age: 64 years). Received the treatment described. Distribution of the ages is displayed in Figure 1, and the correlation between age and the GOS grades is shown in Table 1.

\begin{tabular}{|l|l|l|l|l|l|}
\hline \multicolumn{6}{|l|}{ Distribution of age to GOS } \\
\hline AGE & GOS 1 & GOS 2 & GOS 3 & GOS 4 & GOS 5 \\
\hline$<50 y$ & 0 & 0 & 1 & 0 & 1 \\
\hline $51-60 y$ & 1 & 1 & 5 & 1 & 0 \\
\hline $61-70 y$ & 0 & 0 & 0 & 1 & 2 \\
\hline$>70 y$ & 2 & 1 & 4 & 2 & 0 \\
\hline
\end{tabular}

Table 1: Distribution of age in relation to GOS

On the basis of the GCS classification, 7 patients (31.8\%) were classified as in a poor clinical condition, 11 patients (50\%) in intermediate and 4 patients (18.2\%) in a good clinical condition. The total mortality was calculated with $n=3$ (13.64\%). According to the summarized outcome groups based on GOS score, 5 patients had a poor outcome $(22.7 \%), 10$ patients (45.5) an intermediate outcome and 7 patients (31.8\%) achieved a good outcome. The relation of GCS to GOS is displayed in Table 2.

\begin{tabular}{|l|l|l|l|l|}
\hline \multicolumn{5}{|l|}{ Relation of GCS to GOS } \\
\hline GCS/GOS & N & Poor & Intermediate & Good \\
\hline Poor & 7 & 1 & 6 & 0 \\
\hline Intermediate & 11 & 3 & 4 & 4 \\
\hline Good & 4 & 1 & 0 & 3 \\
\hline
\end{tabular}

Table 2: Relation of GCS to GOS

The mean volume of hemorrhage of all patients before treatment was $35.14 \mathrm{~mL}$ (range $11.5 \mathrm{~mL}-67.15 \mathrm{~mL}$ ). Mean volume after first injection was $22.45 \mathrm{~mL}$ (reduction by $36.1 \%$ ), after second injection $15.93 \mathrm{~mL}$ (reduction by $54.7 \%$ ) and after third injection $10.88 \mathrm{~mL}$ (reduction by $73.05 \%$ ). The blood volume measurements for all patients at all-time points are detailed in Table 3.

\begin{tabular}{|l|l|l|l|l|l|}
\hline \multicolumn{5}{|l|}{ Size of Hemorrhage } \\
\hline Patient & Initial Vol. & 1st & 2nd & 3rd & Total (\%) \\
\hline 1 & 11.547 & 9.468 & 6.282 & 2.177 & 81.14 \\
\hline 2 & 18.292 & 16.408 & 5.292 & 1.768 & 90.33 \\
\hline 3 & 19.667 & 13.870 & 9.302 & 2.860 & 85.45 \\
\hline 4 & 23.047 & 7.894 & 3.399 & 0 & 100.00 \\
\hline
\end{tabular}


Citation: Ntoulias G, Maslehaty H, Petridis AK, Bitter A, Morina D, et al. (2014) Basal Ganglia Hemorrhage: Minimally Invasive and NeuroNavigation Guided Treatment by Injection of Recombinant Tissue-Type Plasminogen Activator (rtPA). J Neurol Disord 2: 158. doi: $10.4172 / 2329-6895.1000158$

Page 3 of 5

\begin{tabular}{|l|l|l|l|l|l|}
\hline 5 & 35.761 & 26.691 & 18.174 & 12.453 & 65.17 \\
\hline 6 & 24.883 & 20.062 & 13.692 & 4.867 & 80.44 \\
\hline 7 & 25.806 & 14.250 & 7.486 & 5.418 & 79.00 \\
\hline 8 & 32.393 & 24.052 & 18.359 & 11.648 & 64.04 \\
\hline 9 & 37.346 & 47.520 & 2.813 & 0 & 100.00 \\
\hline 10 & 45.508 & 22.962 & 9.096 & 7.178 & 84.22 \\
\hline 11 & 48.752 & 40.121 & 48.279 & 45.372 & 6.93 \\
\hline 12 & 57.252 & 19.776 & 6.175 & 3.932 & 93.13 \\
\hline 13 & 60.043 & 45.556 & 30.224 & 26.928 & 55.15 \\
\hline 14 & 67.161 & 34.753 & 21.050 & 14.118 & 78.97 \\
\hline 15 & 17.561 & 14.430 & 6.132 & 4.145 & 76.39 \\
\hline 16 & 19.537 & 9.616 & 27.442 & 15.934 & 18.44 \\
\hline 17 & 28.074 & 9.471 & 5.373 & 5.069 & 81.94 \\
\hline 18 & 30.762 & 15.831 & 13.445 & 7.885 & 74.36 \\
\hline 19 & 34.484 & 16.596 & 33.294 & 28.567 & 17.15 \\
\hline 20 & 41.456 & 33.818 & 12.226 & 2.014 & 93.10 \\
\hline 21 & 45.992 & 48.694 & 35.196 & 26.367 & 42.67 \\
\hline 22 & 47.655 & 2.115 & 17.800 & 11.024 & 76.86 \\
\hline Mean & 35.135 & 22.452 & 15.933 & 10.896 & 73.05 \\
\hline
\end{tabular}

of the drainage was confirmed by CT scanning after first injection in all cases $(100 \%)$.

Two exemplary cases are displayed in Figures $2 \mathrm{a}$ and $2 \mathrm{~b}$ to demonstrate the localization of hemorrhage and catheter, as well as the results of the therapy.

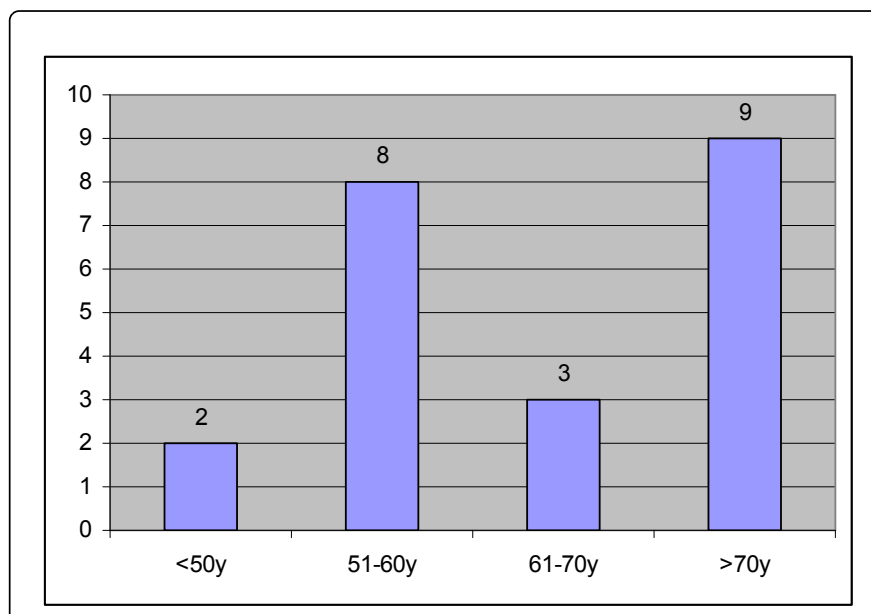

Figure 1: Age Distribution

Table 3: Hemorrhage size; Initial volume in $\mathrm{mL}$; 1st: Volume after first injection, 2nd: Volume after second injection, 3rd: Volume after third injection, total: total percentage of hemorrhage reduction

On the basis of our four defined sub-groups of hemorrhage reduction, 14 patients $(63.6 \%)$ had a total volume reduction $>75 \%, 4$ patients $(18.2 \%) 51-75 \%, 1$ patient $(0.5 \%) 25-50 \%$ and 3 patients (1.5\%) volume reduction $<25 \%$. The correlation of volume reduction with GOS is displayed in Table 4.

\begin{tabular}{|l|l|l|l|l|}
\hline \multicolumn{6}{|l|}{ Relation of Volume reduction to GOS } \\
\hline Vol./GOS & N & Poor & Intermediate & Good \\
\hline$<25 \%$ & 3 & 1 & 2 & 0 \\
\hline $25-50 \%$ & 1 & 1 & 0 & 0 \\
\hline $51-75 \%$ & 4 & 0 & 3 & 1 \\
\hline$>75 \%$ & 14 & 3 & 5 & 6 \\
\hline
\end{tabular}

Table 4: Relation of Volume reduction to GOS

Re-bleeding occurred in 4 cases (18.2\%; Table 2: cases 9, 11, 19 and 21). Despite the break of trPA Lysis in these cases volume reduction could be reached in $100 \%$ in case $9,6.9 \%$ in case $11,17.15 \%$ in case 19 and $42.67 \%$ in case 21 . Since the blood pressure of the patients were monitored in the ICU and treated adequately, an episode of hypertensive peaks was not observed. However, elevated blood pressure was the most important cause of BGH. The correct placement

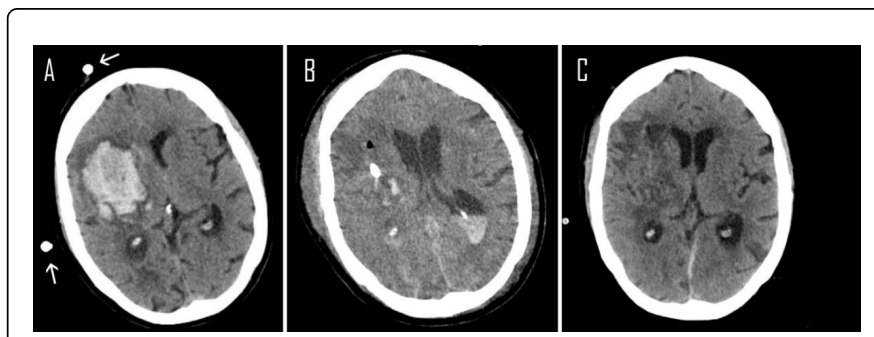

Figure 2a: CT scan of patient 1. A indicates initial CT scan at the time of admission B indicates CT scan following the 1st rtPA injection in which the catheter is visible, $\mathrm{C}$ indicates CT scan following the 3rd rtPA injection. The arrows indicate the markers of the guidance (Brain Lab)

\section{Discussion}

The results of our trial show that injection of rt-PA is an easy and safe treatment for spontaneous BGH. The procedure was performed without major problems and with very good results regarding the correct placement of the catheter under image-guidance. Thus, this technique can be easily performed even by inexperienced neurosurgeons-i.e. by advanced residents. Furthermore, this surgical method was very effective in terms of reduction of the hematoma size. After 3 days of treatment, we observed a mean volume reduction of $73.05 \%$. Compared to our previous experience and the data reported in the literature concerning conservatively treated patients with $\mathrm{ICH}$ or BGH, this method clearly precipitates the clot lysis process. For that reason we dispensed from presenting a control group, because this is not the issue to show, whether clot lysis is faster with rt-PA than without. Further prospective and randomized studies which include homogeneous groups of patients with similar size of hemorrhage, age and clinical condition as a control would better determine whether 
Citation: Ntoulias G, Maslehaty H, Petridis AK, Bitter A, Morina D, et al. (2014) Basal Ganglia Hemorrhage: Minimally Invasive and NeuroNavigation Guided Treatment by Injection of Recombinant Tissue-Type Plasminogen Activator (rtPA). J Neurol Disord 2: 158. doi: $10.4172 / 2329-6895.1000158$

Page 4 of 5

this technique can improve the outcome of patients with spontaneous BGH.

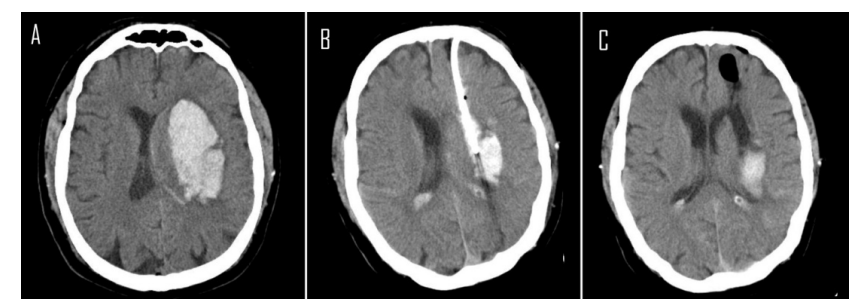

Figure 2b: CT scan of patient 2. A indicates the initial CT scan, B indicates the patient CT scan following the 2nd rtPA injection. Although the space occupying effect after the 2nd rtPA injection is extremely reduced, one more rtPA injection for was needed, $\mathrm{C}$ indicates the patient CT scan following the 3rd rtPA injection. There is no complete clearance of the hematoma, because of a lack of space occupying effect there was no need for an open evacuation. Statistical analysis referring GCS, GOS and volume reduction of the hemorrhage did not show a significant result $(\mathrm{p}=0.0877)$.

As observed in our study, rt-PA injection may carry a risk of rebleeding, which occurred in 4 of the cases examined (18.2\%). Except for one case, with total volume reduction of $100 \%$, the remaining cases of re-bleeding resulted in a poor or intermediate outcome. However, the total volume of hemorrhage as a result of re-bleeding did not exceed the initial volume size significantly, so that the poor outcome of the patients referred to the initial volume size in combination with the clinical condition at time of admission.

Despite the lack of statistical significance due to the relative small number of cases examined, the clinical value of this method and its influence on the clinical course and outcome for this type of patient should be discussed.

It is widely recognized that early removal of a hemorrhage has a positive effect on brain recovery. This effect is caused by the reduction of intracranial pressure and edema. Hereby the brain perfusion is improved and the toxicity of blood breakdown products is reduced $(17,23,25,26,28]$.

However, it seems that clot lysis alone is not the only predictive factor for a better outcome in these patients. Other co-factors such as age, clinical condition of the patients at time of admission, HTN, anticoagulant therapy, metabolic syndrome seem to play an important role and influence the outcome in our study.

Tsai et al. [24] studied 199 patients with BGH and concluded that advanced age and clinical condition at the time of admission were independent predictors.

Comparably to our study, different minimally invasive therapeutical approaches have focused on the treatment of deep seated ICH. For instance, Zhou et al. [30] compared the minimally invasive stereotactic puncture therapy of ICH with conventional craniotomy in a prospective controlled study. The authors included 90 patients in the stereotactic and 78 in the craniotomy arm and found that stereotactic puncture of deep seated ICH could efficiently clear the hemorrhage in a very safe manner, could prevent and relieve hydrocephalus, reduce the intracranial hypertension and relieve the cytotoxicity of blood breakdown products. Hence, the authors concluded that stereotactic puncture of ICH might be superior to conventional craniotomy with a trend towards improved long-term outcome.

Wang et al. [27] compared the minimally invasive cranio-puncture therapy with conservative treatment of $\mathrm{BGH}$ of volumes between 25-40 mL. In their prospective multi-centre study, the authors included 195 patients in the surgical and 182 patients in the conservative arm. The authors concluded that the outcome of the surgical group was significantly better compared to the control group. At the end of the third month, there was a significant difference between the two groups in their ability to conduct activities of daily living, without differences in mortality.

A similar study is presented by Zheng et al. [29]. The authors analysed the data for 595 patients with BGH between $25-35 \mathrm{~mL}$ and compared cranio-puncture to conservative therapy. The conclusion was that cranio-puncture performed 6-48 hours after onset had a benefit compared to conservative therapy.

Furthermore, an on-going prospective trial by Zan et al. [28] is examining the effect of endoscopic surgery compared to conventional craniotomy for BGH. Another recently published work by Dye et al. [5] focused on image-guided endoscopic evacuation of $\mathrm{ICH}$ via a frontal burr hole through an eyebrow incision. In their study, performed on six patients, the authors reported a mean reduction of hemorrhage by $79.2 \%$ (range $49 \%-92.7 \%$ ), suggesting that this procedure might be useful for anterior BGH.

With regards to the application of rt-PA for clot lysis in deep seated $\mathrm{ICH}$, the prospective trial Stereotactic Treatment of Intracerebral Hematoma by Means of a Plasminogen Activator (SICHPA) compared to conservative treatment by Teernstra et al. [23] should also be discussed. The authors included patients with $\mathrm{ICH}$ in different locations. Despite the proven significant volume reduction in the surgical arm, a significant difference in overall survival in three months could not be observed [23]. Lippitz et al. [13] injected rtPA to resolve the remaining blood clots after stereotactic aspiration of BGH. Since their encouraging results with an average clot lysis of $84 \%$, the authors came to the conclusion that the application of local rtPA is an effective additional treatment method for further resolution of deep seated ICH after stereotactic aspiration.

Alternatively to minimally invasive surgical models for deep seated $\mathrm{ICH}$, some authors propose a maximally invasive procedure in terms of decompressive hemicraniectomy, followed by open surgical removal of ICH $[1,14]$.

Ma et al. [14] show that decompressive craniectomy in addition to hematoma evacuation might reduce the mortality in patients suffering from BGH compared to conservative treatment.

As we can see, many of these studies aim to improve the outcome in patients suffering from BGH. According to our results and the recent literature, minimally invasive treatment strategies have the potential to improve the clinical course and outcome in this particular group of patients. Whether the treatment of BGH with rt-PA is superior to other treatments will be addressed in future and larger prospective studies.

\section{Conclusion}

Injection of rt-PA for BGH treatment seems an easy and safe minimally invasive alternative to open surgical hematoma evacuation and might be more favorable compared to conservative treatment. In 
Citation: Ntoulias G, Maslehaty H, Petridis AK, Bitter A, Morina D, et al. (2014) Basal Ganglia Hemorrhage: Minimally Invasive and NeuroNavigation Guided Treatment by Injection of Recombinant Tissue-Type Plasminogen Activator (rtPA). J Neurol Disord 2: 158. doi: $10.4172 / 2329-6895.1000158$

Page 5 of 5

our study we observed a mean volume reduction of $73 \%$ after 3 days of treatment with rtPA injection in 22 patients. However, clot lysis alone is not the only predictive factor for a better outcome. Outcome prognosis should take into account several additional co-factors. Large prospective and randomized trials are necessary to address many open questions.

\section{References}

1. Arméstar F, Moreno JA, Cladella JM, Puyalto P (2010) Hemorrhagic stroke in basal ganglia treated with decompressive hemicraniectomy. Rev Neurol 50: 511.

2. Broderick J, Brott T, Tomsick T, Leach A (1993) Lobar hemorrhage in the elderly. The undiminishing importance of hypertension. Stroke 24: 49-51.

3. Broderick JP, Brott TG, Duldner JE, Tomsick T, Huster G (1993) Volume of intracerebral hemorrhage. A powerful and easy-to-use predictor of 30day mortality. Stroke 24: 987-993.

4. Diamond P, Gale S, Stewart K (2003) Primary intracerebral haemorrhage-clinical and radiologic predictors of survival and functional outcome. Disabil Rehabil 25: 689-698.

5. Dye JA, Dusick JR, Lee DJ, Gonzalez NR, Martin NA (2012) Frontal bur hole through an eyebrow incision for image-guided endoscopic evacuation of spontaneous intracerebral hemorrhage. J Neurosurg 117: 767-773.

6. Fogelholm R, Nuutila M, Vuorela AL (1992) Primary intracerebral haemorrhage in the Jyvaskyla region, central Finland, 1985-89: incidence, case fatality rate, and functional outcome. J Neurol Neurosurg Psychiatry 55: 546-552.

7. Foulkes MA, Wolf PA, Price TR, Mohr JP, Hier DB (1988) The Stroke Data Bank: design, methods, and baseline characteristics. Stroke 19: 547-554.

8. Hai J, Zhang L, Wang F, Wan JF, Pan QG (2010) Quality of life with special respect to depression after surgical treatment of hypertensive basal ganglia hemorrhage. Neurol India 58: 74-77.

9. Hattori N, Katayama Y, Maya Y, Gatherer A (2004) Impact of stereotactic hematoma evacuation on activities of daily living during the chronic period following spontaneous putaminal hemorrhage: a randomized study. J Neurosurg 101: 417-420.

10. Kalff R, Feldges A, Mehdorn HM, Grote W (1992) Spontaneus intracerebral hemorrhage. Neurosurg Rev 15: 177-186.

11. Kirkman MA, Mahattanakul W, Gregson BA, Mendelow AD (2008) The effect of the results of the STICH trial on the management of spontaneous supratentorial intracerebral haemorrhage in Newcastle. Br J Neurosurg 22: 739-746.

12. Kothari RU, Brott T, Broderick JP, Barsan WG, Sauerbeck LR, et al. (1996) The ABCs of measuring intracerebral hemorrhage volumes. Stroke 27: 1304-1305.

13. Lippitz BE, Mayfrank L, Spetzger U, Warnke JP, Bertalanffy H, et al. (1994) Lysis of basal ganglia haematoma with recombinant tissue plasminogen activator (rtPA) after stereotactic aspiration: initial results. ActaNeurochir (Wien) 127: 157-160.

14. Ma L, Liu WG, Sheng HS, Fan J, Hu WW, et al. (2010) Decompressive craniectomy in addition to hematoma evacuation improves mortality of patients with spontaneous basal ganglia hemorrhage. J Stroke Cerebrovasc Dis 19: 294-298.

15. Maslehaty H, Petridis AK, Barth H, Doukas A, Mehdorn HM (2012) Treatment of 817 patients with spontaneous supratentorial intracerebral hemorrhage: characteristics, predictive factors and outcome. Clin Pract 2: e56.
16. Mendelow AD, Gregson BA, Fernandes HM, Murray GD, Teasdale GM, et al. (2005) Early surgery versus initial conservative treatment in patients with spontaneous supratentorial intracerebral haematomas in the International Surgical Trial in Intracerebral Haemorrhage (STICH): a randomised trial. Lancet 365: 387-397.

17. Miller DW, Barnett GH, Kormos DW, Steiner CP (1993) Stereotactically guided thrombolysis of deep cerebral hemorrhage: preliminary results. Cleve Clin J Med 60: 321-324.

18. Nakano T, Ohkuma H (2005) Surgery versus conservative treatment for intracerebral haemorrhage-is there an end to the long controversy? Lancet 365: 361-362.

19. Schaller C, Rohde V, Meyer B, Hassler W (1995) Stereotactic puncture and lysis of spontaneous intracerebral hemorrhage using recombinant tissue-plasminogen activator. Neurosurgery 36: 328-333.

20. Schwarz S, Häfner K, Aschoff A, Schwab S (2000) Incidence and prognostic significance of fever following intracerebral hemorrhage. Neurology 54: 354-361.

21. Talacchi A, Ricci UM, Caramia G, Massimo G (2011) Basal ganglia haemorrhages: efficacy and limits of different surgical strategies. $\mathrm{Br} \mathrm{J}$ Neurosurg 25: 235-242.

22. Tatu L, Moulin T, El Mohamad R, Vuillier F, Rumbach L, et al. (2000) Primary intracerebral hemorrhages in the Besancon stroke registry. Initial clinical and CT findings, early course and 30-day outcome in 350 patients. EurNeurol 43: 209-214.

23. Teernstra OP, Evers SM, Lodder J, Leffers P, Franke CL, et al. (2003) Stereotactic treatment of intracerebral hematoma by means of a plasminogen activator: a multicenter randomized controlled trial (SICHPA). Stroke 34: 968-974.

24. Tsai JP, Hung CL, Ku SL, Hou CJ, Yeh HI, et al. (2011) Factors influencing therapeutic strategy for patients with basal ganglia hemorrhage-could age play a potential role in final treatment decision? Acta Neurol Belg 111: 268-275.

25. Vespa P, McArthur D, Miller C, O'Phelan K, Frazee J, et al. (2005) Frameless stereotactic aspiration and thrombolysis of deep intracerebral hemorrhage is associated with reduction of hemorrhage volume and neurological improvement. Neurocrit Care 2: 274-281.

26. Wagner KR, Xi GH, Hua Y, Zuccarello M, de Courten-Myers GM, et al. (1999) Ultra-early clot aspiration after lysis with tissue plasminogen activator in a porcine model of intracerebral hemorrhage: edema reduction and blood-brain barrier protection. J Neurosurg 90: 491-498.

27. Wang WZ, Jiang B, Liu HM, Li D, Lu CZ, et al. (2009) Minimally invasive craniopuncture therapy vs. conservative treatment for spontaneous intracerebral hemorrhage: results from a randomized clinical trial in China. International journal of stroke 4: 11-16.

28. Zan X, Li H, Liu W, Fang Y, Ma J, et al. (2012) Endoscopic surgery versus conservative treatment for the moderate-volume hematoma in spontaneous basal ganglia hemorrhage (ECMOH): study protocol for a randomized controlled trial. BMC Neurol 12: 34.

29. Zheng W, Zhang C, Hou D, Cao C (2012) Comparison on different strategies for treatments of hypertensive hemorrhage in the basal ganglia region with a volume of 25 to $35 \mathrm{ml}$. Acta Cir Bras 27: 727-731.

30. Zhou H, Zhang Y, Liu L, Han X, Tao Y, et al. (2011) A prospective controlled study: minimally invasive stereotactic puncture therapy versus conventional craniotomy in the treatment of acute intracerebral hemorrhage. BMC Neurol 11: 76.

31. Zuo Y, Cheng G, Gao DK, Zhang X, Zhen HN, et al. (2009) Gross-total hematoma removal of hypertensive basal ganglia hemorrhages: a longterm follow-up. J NeurolSci 287: 100-104. 